\title{
Night Blindness, CTCAE
}

National Cancer Institute

\section{Source}

National Cancer Institute. Night Blindness, CT CAE. NCI Thesaurus. Code C143705.

A disorder characterized by an inability to see clearly in dim light. 